\title{
JACOBIANS OF SINGULAR SPECTRAL CURVES AND COMPLETELY INTEGRABLE SYSTEMS
}

\author{
OLIVIER VIVOLO \\ Laboratoire Emile Picard, URA CNRS 5580, Université Paul Sabatier, \\ 118 Route de Narbonne, 31062 Toulouse Cedex, France \\ (vivolo@picard.ups-tlse.fr)
}

(Received 2 November 1998)

\begin{abstract}
Consider an isospectral manifold formed by matrices $M \in \mathfrak{g l}_{r}(\mathbb{C})[x]$ with a fixed leading term. The description of such a manifold is well known in the case of a diagonal leading term with different eigenvalues. On the other hand, there are many important systems where this term has multiple eigenvalues. One approach is to impose conditions in the sub-leading term. The result is that the isospectral set is a smooth manifold, bi-holomorphic to a Zariski open subset of the generalized Jacobian of a singular curve.
\end{abstract}

Keywords: integrability; Jacobian variety

AMS 2000 Mathematics subject classification: Primary 37J35; 70H06

\section{Introduction}

Consider a Hamiltonian system written in the form of a Lax pair, i.e. (d/dt) $A(x)=$ $[A(x), B(x)]$, where $A(x)$ and $B(x)$ are complex matrix polynomials in a variable $x$, i.e.

$$
\begin{aligned}
& A(x)=A_{d} x^{d}+A_{d-1} x^{d-1}+\cdots+A_{0}, \quad A_{i} \in \mathfrak{g l}_{r}(\mathbb{C}), \\
& B(x)=B_{l} x^{l}+B_{l-1} x^{l-1}+\cdots+B_{0}, \quad B_{i} \in \mathfrak{g l}_{r}(\mathbb{C}) .
\end{aligned}
$$

The coefficients $A_{i}, B_{j}$ are matrices which depend on the dynamical variables. The coefficients of the spectral polynomial $P(x, y)=\operatorname{det}\left(A(x)-y \operatorname{Id}_{r}\right)$ are the first integrals of the system. It is easy to see that the matrix $A_{d}$ is invariant of time, consequently it is natural to fix it. Denote by $M_{P}^{J}$ the isospectral variety

$$
M_{P}^{J}=\left\{A(x): \operatorname{det}\left(A(x)-y \operatorname{Id}_{r}\right)=P(x, y)=0 \text { and } A_{d}=J\right\} .
$$

The affine curve $X_{\mathrm{a}}=\left\{(x, y) \in \mathbb{C}^{2}: P(x, y)=0\right\}$ is embedded in $\mathcal{O}_{3}(d)$. Let $\bar{X}_{\mathrm{a}}$ be the compactification of $X_{\mathrm{a}}$, we will call it the spectral curve associated to the matrix polynomial $A(x)$. If the affine curve $X_{\mathrm{a}}$ is smooth, then it is easy to check that the variety $M_{P}^{J}$ is smooth (L. Gavrilov, personal communication; see also [8]). 

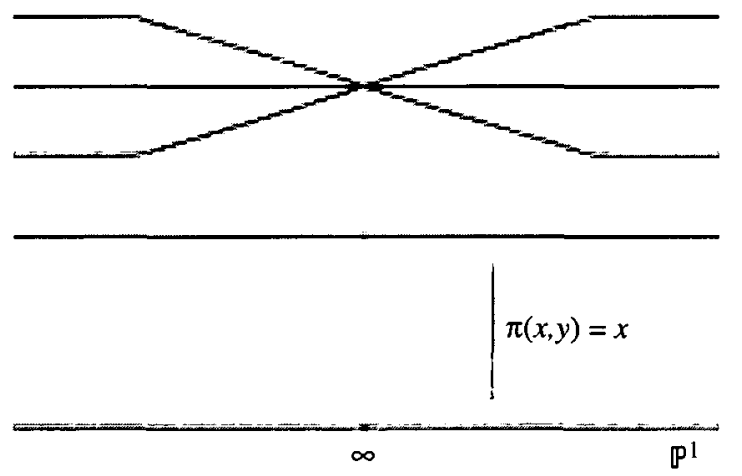

Figure 1 . Spectral curve with an ordinary 3-tuple point at infinity.

Several articles on the same topic have appeared. Adler and van Moerbeke [2] fixed the matrix $A_{d}=J$ and searched to calculate the variety $M_{P}^{J} / \mathbb{P} G l_{r}(\mathbb{C}, J)\left(\mathbb{P} G l_{r}(\mathbb{C}, J)\right.$ corresponds to a subgroup of the projective group $\mathbb{P} G l_{r}(\mathbb{C})$ formed by matrices which commute with $J$; moreover, this subgroup acts on $M_{P}^{J}$ by conjugation) when the matrix $J$ is diagonal with simple eigenvalues. So the spectral curve becomes smooth (even at infinity). However, it is interesting to consider singularities at infinity, because a lot of integrable systems possess an affine smooth spectral curve and at infinity this curve is singular (this happens when the matrix $J$ has eigenspace, the dimension of which is greater than one), for instance the Kowalevskaya top [5], the anharmonic oscillator, the Garnier system and integrable quartic [14, p. 172].

If the affine curve $X_{\mathrm{a}}$ is smooth, van Moerbeke and Mumford [18] describe the quotient variety $M_{P}^{J} / \mathbb{P} G l_{r}(\mathbb{C}, J)$ as a Zariski open subset in the Jacobian $J(X)$. If the affine curve $X_{\mathrm{a}}$ is not smooth, they express the variety $M_{P}^{J} / \mathbb{P} G l_{r}(\mathbb{C}, J)$ as a Zariski open subset in a generalized Jacobian. The difference between the present paper and [18] is that we describe $M_{P}^{J}$ (not the quotient variety $M_{P}^{J} / \mathbb{P} G l_{r}(\mathbb{C}, J)$ ), and, furthermore, the affine curve $X_{\mathrm{a}}$ is smooth but its compactification is singular at infinity.

The article of Adams, Harnad and Hurtubise [1] gives an expression of the same quotient variety $M_{P}^{J} / \mathbb{P} G l_{r}(\mathbb{C}, J)$ as an affine part of the usual Jacobian $J(X)$. This result is also proved by Beauville [3].

In $[\mathbf{6}, \mathbf{9}, \mathbf{1 3}]$, the authors describe abstract Hamiltonian systems. On the other hand, Hitchin [9] noted that it is important to explicitly find the Hamiltonian differential equations describing these systems. Here, we consider concrete realization of integrable systems. The difference between our systems and the integrable systems of Hitchin type described in the literature $[\mathbf{6}, \mathbf{1 3}]$ is the line bundles, which we consider, are neither stable nor semi-stable; moreover, Hitchin's systems linearize on abelian varieties but our systems linearize on generalized Jacobians.

To conclude, we mention a recent article of Gavrilov [8], who uses smooth spectral curves and shows that the variety $M_{P}^{J}$ is an open subset of a commutative algebraic group (the generalized Jacobian of $\bar{X}_{\mathrm{a}}$ ). 
The purpose of the present article is to describe the variety $M_{P}^{J}$ in the case where the matrix $J$ is diagonalizable and has multiple eigenvalues (then the matrix $J$ is not regular).*

Suppose that the affine curve $X_{\mathrm{a}}$ is smooth and at $Q_{i} \in \bar{X}_{\mathrm{a}}$ (where $x\left(Q_{i}\right)=\infty$ ), $\bar{X}_{\mathrm{a}}$ is locally a normal crossing of many branches. Let $\mathcal{F}=\left\{\left(\mathbb{C}^{r}, J\right),(E, K)\right\}$ be the data at infinity of $\bar{X}_{\text {a }}\left(J\right.$ is an endomorphism $J \in \operatorname{End}\left(\mathbb{C}^{r}\right), E$ a vector space and $K \in \operatorname{End}(E)$, see Definition 3.2. We assume that $J$ is diagonalizable and $K$ is diagonalizable with distinct eigenvalues. Let $X$ be the smooth compactification of $\bar{X}_{\mathrm{a}}$. Let $P_{1}, P_{2}, \ldots, P_{r}$ be the 'infinite' points of the curve $X$ and $X^{\prime} \sim\left(X, \sum_{i} P_{i}\right)$ the singular curve obtained from $X$ identifying the points $P_{1}, P_{2}, \ldots, P_{r}$ with a single point noted $\infty$. Define

$$
M_{P}^{\mathcal{F}}=\left\{A(x) \in M_{P}^{J}:\left.\operatorname{pr}_{E} \cdot A_{d-1}\right|_{E}=K\right\}
$$

where $\mathrm{pr}_{E}$ is the projection on $E$ and $\left.\right|_{E}$ is the restriction to $E$. Denote further by $\Theta^{\prime}$ the canonical Riemann theta divisor formed by special line bundles on $X^{\prime}$ of degree $p_{\mathrm{a}}$ (the arithmetic genus of $X^{\prime}$ ), that is to say $\operatorname{dim} H^{1}\left(X^{\prime}, L^{\prime}\right) \neq 0$.

The main result of this article is the following theorem.

Theorem 1.1. The variety $M_{P}^{\mathcal{F}}$ is smooth and bi-holomorphic to a Zariski open subset of generalized Jacobian of $X^{\prime}, J\left(X^{\prime}\right)-\Theta^{\prime}$.

\section{The generalized Jacobian}

Now, we introduce the notation of this article [17]. The construction and properties of generalized Jacobians are due to Rosenlicht $[\mathbf{1 5}, \mathbf{1 6}]$ (even if the generalized Jacobian has already been used by Jacobi [10]) and Lang $[\mathbf{1 1}, \mathbf{1 2}]$; they rely on the theory of abelian varieties, developed by Weil [20]. We consider $X$ to be a smooth compact irreducible algebraic curve. Let $P_{i}$ for $1 \leqslant i \leqslant s$ be some distinct points on $X$. We put $\mathrm{m}=\sum_{i=1}^{s} n_{i} P_{i}$ and $n_{i} \in \mathbb{N}^{*}, \forall i=1,2, \ldots, s$. We call $S(D)$ the support of some divisor $D$ on $X$, i.e. $S(\mathfrak{m})=\left\{P_{1}, P_{2}, \ldots, P_{s}\right\}$, denote $X_{\text {reg }}=X-S(\mathfrak{m})$ and $r=\sum_{i=1}^{s} n_{i}$.

Definition 2.1. To the pair $(X, \mathrm{~m})$ we associate a singular curve $X^{\prime}=X_{\text {reg }} \cup \infty$. The curve $X^{\prime}$ is, as a topological space, just $X$ with its points $P_{1}, \ldots, P_{s}$ identified with a single point $\infty$. The structure sheaf $\mathcal{O}^{\prime}$ of $X^{\prime}$ is defined in the following way. Let $\mathcal{O}_{X^{\prime}}$ be the direct image of the structure sheaf $\mathcal{O}_{X}$ under canonical projection $X \rightarrow X^{\prime}$. Then

$$
\mathcal{O}_{P}^{\prime}= \begin{cases}\mathcal{O}_{P}, & \text { if } P \in X_{\text {reg }} \\ \mathbb{C}+i_{\infty}, & \text { if } P=\infty\end{cases}
$$

where $i_{\infty}$ is the ideal of $\mathcal{O}_{\infty}$ formed by the functions $f$ having a zero at $P_{i}$ of order at least $n_{i}$.

We define the sheaf $\mathcal{L}^{\prime}(D)$, where $D$ is a divisor on $X$ such that $S(D) \bigcap S(\mathrm{~m})=\emptyset$, by

$$
\mathcal{L}^{\prime}(D)_{P}= \begin{cases}\mathcal{L}(D)_{P}, & \text { if } P \in X_{\mathrm{reg}} \\ \mathcal{O}_{\infty}^{\prime}, & \text { if } P=\infty\end{cases}
$$

* A matrix is regular if all eigenspaces are of dimension one. 
The sheaf $\mathcal{L}^{\prime}(D)$ is a locally free $\mathcal{O}^{\prime}$-module of rank one and there is a one-to-one correspondence between isomorphism classes of invertible sheaves $\mathcal{L}^{\prime}$ on $X^{\prime}$ and isomorphism classes of line bundles $L^{\prime}$ over $X^{\prime}$.

Let

$$
\begin{aligned}
L^{\prime}(D) & =H^{0}\left(X^{\prime}, \mathcal{L}^{\prime}(D)\right), & I^{\prime}(D) & =H^{1}\left(X^{\prime}, \mathcal{L}^{\prime}(D)\right) \\
l^{\prime}(D) & =\operatorname{dim}_{\mathbb{C}} L^{\prime}(D), & i^{\prime}(D) & =\operatorname{dim}_{\mathbb{C}} I^{\prime}(D)
\end{aligned}
$$

As the sheaf $\mathcal{O}_{X} / \mathcal{O}_{X}^{\prime}$ is coherent, we put $\delta_{P}=\operatorname{dim}_{\mathbb{C}}\left(\mathcal{O}_{P} / \mathcal{O}_{P}^{\prime}\right)$ with $P \in X^{\prime}$, the arithmetic genus $p_{\mathrm{a}}$ (dimension of $H^{1}\left(X^{\prime}, \mathcal{O}^{\prime}\right)$ ) of the singular curve $X^{\prime}$ is obtained from the geometric genus $p_{\mathrm{g}}$ of $X$ by the relation

$$
p_{\mathrm{a}}=p_{\mathrm{g}}+\delta_{\infty}
$$

In fact,

$$
\delta_{\infty}=\operatorname{dim}_{\mathbb{C}}\left(\frac{\mathcal{O}_{\infty}}{\mathbb{C}+i_{\infty}}\right)=\operatorname{dim}_{\mathbb{C}}\left(\frac{\mathcal{O}_{\infty}}{i_{\infty}}\right)-1=\operatorname{deg}(\mathfrak{m})-1
$$

then

$$
p_{\mathrm{a}}=p_{\mathrm{g}}+\operatorname{deg}(\mathfrak{m})-1
$$

or

$$
p_{\mathrm{a}}=p_{\mathrm{g}}+r-1 \text {. }
$$

A divisor $D$ on $X$ with $S(D) \bigcap S(\mathfrak{m})=\emptyset$ verifies

$$
l^{\prime}(D)-i^{\prime}(D)=\operatorname{deg}(D)+1-p_{\mathrm{a}}
$$

Now we define the equivalence relation $\stackrel{\mathfrak{m}}{\sim}$.

Definition 2.2. Let $D_{1}$ and $D_{2}$ be two divisors on $X$ with $S\left(D_{1}\right) \bigcap S(\mathfrak{m})=\emptyset$ and $S\left(D_{2}\right) \bigcap S(\mathfrak{m})=\emptyset$. Then $D_{1} \stackrel{\mathfrak{m}}{\sim} D_{2}$, provided that there exists a global meromorphic function $f$ on $X$, such that $(f)=D_{1}-D_{2}$ and $v_{P_{i}}(f-1) \geqslant n_{i}, i=1,2, \ldots, s\left(v_{P}(\cdot)\right.$ is the order function at $P$ ).

Definition 2.3. We call the subgroup $\operatorname{Pic}^{0}\left(X^{\prime}\right)$ of $\operatorname{Pic}\left(X^{\prime}\right):=\operatorname{Div}\left(X^{\prime}\right) / \stackrel{\mathfrak{m}}{\sim}$ formed by the divisors $D$ on $X$ with $S(D) \cap S(\mathrm{~m})=\emptyset$ and $\operatorname{deg}(D)=0$ the generalized Jacobian of $X^{\prime}$, denoted $J\left(X^{\prime}\right)$.

It is known that $J\left(X^{\prime}\right)$ is an extension of $J(X)$, the Jacobian of $X$, by the algebraic group $\left(\mathbb{C}^{*}\right)^{s-1} \times \mathbb{C}^{r-s}$ :

$$
0 \rightarrow\left(\mathbb{C}^{*}\right)^{s-1} \times \mathbb{C}^{r-s} \rightarrow J\left(X^{\prime}\right) \stackrel{\phi}{\rightarrow} J(X) \rightarrow 0
$$

Remark 2.4. There is a one-to-one correspondence between isomorphism classes of invertible sheaves $\mathcal{L}$ on $X^{\prime}$ and isomorphism classes of line bundles $L$ over $X^{\prime}$. 


\section{The spectral curve}

Let $x$ be an affine coordinate on $\mathbb{P}^{1}$. We now consider the vector space $M$ of all complex matrix polynomials $A(x)$ in a variable $x$, of fixed degree $d(d \geqslant 1)$ and dimension $r$ $(r \geqslant 3)$

$$
A(x)=A_{d} x^{d}+A_{d-1} x^{d-1}+\cdots+A_{0}, \quad A_{i} \in \mathfrak{g l}_{r}(\mathbb{C}) .
$$

Let

$$
M_{P}=\left\{A(x) \in M: \operatorname{det}\left(A(x)-y \operatorname{Id}_{r}\right)=P(x, y)=0\right\}
$$

and $M^{J}$ be the affine subspace formed by matrices with a fixed leading coefficient $A_{d}=J$. We put

$$
M_{P}^{J}=M_{P} \cap M^{J}=\left\{A(x) \in M^{J}: \operatorname{det}\left(A(x)-y \operatorname{Id}_{r}\right)=P(x, y)=0\right\},
$$

where $P(x, y)$ is an irreducible fixed polynomial,

$$
P(x, y)=\operatorname{det}\left(A(x)-y \operatorname{Id}_{r}\right)=(-1)^{r} y^{r}+s_{1}(x) y^{r-1}+\cdots+s_{r}(x),
$$

with $\operatorname{deg}\left(s_{i}(x)\right) \leqslant i . d$. Let $\mathbb{P}^{2}(d)$ be the $d$-weighted projective space defined by $\left(\mathbb{C}^{3}-\right.$ $\{(0,0,0)\}) / \sim$, where $(x, y, z) \sim\left(\lambda x, \lambda^{d} y, \lambda z\right)$ with $\lambda \in \mathbb{C}^{*}$.

Definition 3.1. Let $X_{\mathrm{a}}=\left\{(x, y) \in \mathbb{C}^{2}: P(x, y)=0\right\}$ be the curve associated to the irreducible polynomial $P(x, y)$. The spectral curve is the closure $\bar{X}_{\mathrm{a}}$ of $X_{\mathrm{a}}$ in $\mathbb{P}^{2}(d)$.

Now fix a spectral polynomial $P(x, y)=\operatorname{det}(A(x)-y \mathrm{Id})$, and assume that $A_{d}$ is diagonalizable.

Let

$$
E_{d}=\mathbb{C}^{r}, \quad u_{d}=A_{d} .
$$

Let $u_{d} \in \operatorname{End}\left(E_{d}\right)$ be an endomorphism of $E_{d}$ and $S p\left(u_{d}\right)=\left\{\lambda_{1}, \ldots, \lambda_{k}\right\}$ of respectively order $s_{1}, \ldots, s_{k}$, we introduce the set

$$
I_{d}=\left\{i: \operatorname{dim} \operatorname{Ker}\left(u_{d}-\lambda_{i}\right)>1\right\} .
$$

If $I_{d} \neq \emptyset$, then we can define

$$
E_{d-1}:=\bigoplus_{i \in I_{d}} \operatorname{Ker}\left(u_{d}-\lambda_{i}\right)^{s_{i}}
$$

and $u_{d-1} \in \operatorname{End}\left(E_{d-1}\right)$ by

$$
u_{d-1}:=\bigoplus_{i \in I_{d}} u_{d-1}^{i}: E_{d-1} \rightarrow E_{d-1},
$$

where $u_{d-1}^{i}, i \in I_{d}$ is the endomorphism

$$
\begin{aligned}
& u_{d-1}^{i}: \operatorname{Ker}\left(u_{d}-\lambda_{i}\right)^{s_{i}} \rightarrow E_{d} \rightarrow \operatorname{Ker}\left(u_{d}-\lambda_{i}\right)^{s_{i}} \\
& v \mapsto A_{d-1}(v) \mapsto \operatorname{pr}\left(A_{d-1}(v)\right)
\end{aligned}
$$


Definition 3.2. The data $\mathcal{F}$ of the spectral curve at infinity is the set

$$
\mathcal{F}:=\left\{\left(E_{d}, u_{d}\right),\left(E_{d-1}, u_{d-1}\right)\right\} .
$$

Definition 3.3. The projective sub-group of $\mathbb{P} G l_{r}(\mathbb{C})$ such that the data $\mathcal{F}$ is invariant under the conjugation action of this sub-group is denoted $\mathbb{P} G l_{r}(\mathbb{C} ; \mathcal{F})$

$$
\mathbb{P} G l_{r}(\mathbb{C} ; \mathcal{F})=\left\{R \in \mathbb{P} G l_{r}(\mathbb{C}):\left.\forall k \in\{d, d-1\} R_{E_{k}} u_{k}\right|_{E_{k}}=u_{k}\right\}
$$

and

$$
\begin{aligned}
& R_{E_{k}}: E_{k} \rightarrow E_{d} \rightarrow E_{k} \\
& v \mapsto R v R^{-1} \mapsto \operatorname{pr}\left(R v R^{-1}\right) .
\end{aligned}
$$

We assume that the curve $\bar{X}_{\mathrm{a}}-\sum_{i \in I_{d}} Q_{i}$ is smooth (where $x\left(Q_{i}\right)=\infty$ and $y\left(Q_{i}\right)=\lambda_{i}$ ) and, at $Q_{i}, \bar{X}_{\mathrm{a}}$ is a normal crossing of $s_{i}$ branches. Let $\mathcal{F}:=\left\{\left(E_{d}, u_{d}\right),\left(E_{d-1}, u_{d-1}\right)\right\}$ be the data of the spectral curve at infinity where $u_{d}$ is diagonalizable and $u_{d-1}$ is diagonalizable with distinct eigenvalues. The geometric genus of $\bar{X}_{\mathrm{a}}$ is

$$
p_{\mathrm{g}}=\frac{1}{2}(r-1)(r d-2)-\sum_{i \in I_{d}} \delta_{Q_{i}}=\frac{1}{2}(r-1)(r d-2)-\sum_{i \in I_{d}} \frac{1}{2} s_{i}\left(s_{i}-1\right) .
$$

Let $X$ be the smooth compactification of $\bar{X}_{\mathrm{a}}$. When we desingularize $\bar{X}_{\mathrm{a}}$, each point $Q_{i}$ gives $s_{i}$ distinct points on $X$.

The projection

$$
\begin{aligned}
\pi: \mathbb{P}^{2}(d)-\{[0,1,0]\} & \rightarrow \mathbb{P}^{1} \\
{[x, y, z] } & \mapsto[x, z]
\end{aligned}
$$

identifies the surface $\mathbb{P}^{2}(d)-\{[0,1,0]\}$ with the holomorphic line bundle $\mathcal{O}_{\mathbb{P}^{1}}(d)$, and, as $[0,1,0] \notin X$, then $X$ is naturally embedded in $\mathcal{O}_{\mathbb{P}^{1}}(d)$ too. The induced projection

$$
\pi: X \rightarrow \mathbb{P}^{1}
$$

is a ramified covering of degree $r$, and over the affine plane $\mathbb{C}$ it is simply the first projection

$$
\begin{aligned}
\pi: X & \rightarrow \mathbb{C} \\
(x, y) & \mapsto x
\end{aligned}
$$

Definition 3.4. If $\pi^{-1}([1,0])=\sum_{i=1}^{r} P_{i}$, then we define the modulus $m$ to be the effective divisor $\mathfrak{m}=\sum_{i=1}^{r} P_{i}$. We denote by $X^{\prime}=X_{\mathfrak{m}}$ the singular curve obtained from $X$ when we identify its points $P_{1}, P_{2}, \ldots, P_{r}$ with a single point $\infty$. The arithmetic genus of $X^{\prime}$ is given by $p_{\mathrm{a}}=p_{\mathrm{g}}+r-1$.

Remark 3.5. Suppose the multiplicity of $\lambda_{i_{0}}$ is greater than 1 and $\left(u_{d-1}\right)^{i_{0}}$ is homologous to

$$
\left(\begin{array}{ccc|c}
\alpha_{1} & & & \\
& \ddots & & * \\
& & \alpha_{s_{i_{0}}} & \\
\hline & * & & *
\end{array}\right),
$$


and the coefficients $\alpha_{1}, \alpha_{2}, \ldots, \alpha_{s_{i_{0}}}$ are fixed and distinct. The coefficient $\alpha_{j}$ is the coefficient of $z$ in the Puiseux expansion of $y$, i.e. $y=\lambda_{i_{0}}+\alpha_{j} z+\cdots$.

Definition 3.6. Let $P(x, y) \in \mathbb{C}[x, y]$ and $\mathcal{F}=\left\{\left(\mathbb{C}^{r}, J\right),(E, K)\right\}$ be the data at infinity of the spectral curve. We introduce the variety $M_{P}^{\mathcal{F}}$ defined by

$$
M_{P}^{\mathcal{F}}=\left\{A(x) \in M_{P}^{J}: A(x)=J x^{d}+A_{d-1} x^{d-1}+\cdots+A_{0},\left.A_{d-1}\right|_{E}=K\right\} .
$$

For $A(x) \in M_{P}^{\mathcal{F}}$ and $R \in \mathbb{P} G l_{r}(\mathbb{C}, \mathcal{F})$, then $R A(x) R^{-1} \in M_{P}^{\mathcal{F}}$, it is easy to see that we can choose a suitable base such that $J$ and $K$ are simultaneously diagonalized.

\section{The eigenvector bundle}

For each non-singular point $p \in X_{\mathrm{a}}$ which is not a branching point of $X$, there is a one-dimensional eigenspace $l(x) \subset \mathbb{C}^{r}$ of $A(x(p))$ with eigenvalue $y(p)$. This gives a holomorphic line bundle on $X_{\mathrm{a}}$ defined everywhere except for singular points and branching points. Let $X$ be the non-singular compact model of $X_{\mathrm{a}}$, the eigenvector bundle extends to a holomorphic line bundle $l$ on the whole smooth curve $X$. Indeed, the mapping $p \rightarrow l(p)$ determines a meromorphic mapping

$$
\begin{aligned}
X & \rightarrow \mathbb{C P}^{r-1} \\
x & \mapsto\left[f_{1}(x), f_{2}(x), \ldots, f_{r}(x)\right] .
\end{aligned}
$$

Since any such mapping is actually holomorphic, the eigenvector bundle extends to $X$. We denote its dual by $L$. If $D$ is the minimal effective divisor such that $\forall i,\left(f_{i}\right) \geqslant-D$, then $L=L_{D}$. If $S(D) \bigcap S(\mathfrak{m}) \neq \emptyset, L_{D}$ does not define a line bundle on $X^{\prime}$, that is why we must normalize the bundle $L_{D}$ to define the eigenvector bundle on $X^{\prime}$.

Proposition 4.1. Let $f(x, y)=\left(f_{1}, f_{2}, \ldots, f_{r}\right)$ be an eigenvector of $A(x) \in M_{P}^{\mathcal{F}}$ normalized by the condition

$$
f_{1}+f_{2}+\cdots+f_{r} \equiv 1 \text {. }
$$

Let $D$ be the minimal divisor, such that $\left(f_{i}\right) \geqslant-D, i=1,2, \ldots, r$. Then we get $S(D) \cap S(\mathrm{~m})=\emptyset$ and we define (the dual to the) eigenvector bundle on the singularized spectral curve $X^{\prime}$.

Proof. If $j \in I_{d}$, we shall write the polynomial $P(x, y, z)$ near the points $Q_{j}=(x=$ $\infty, y=\lambda_{j}$ ). This point give $s_{j}$ distinct points $P_{n_{1}}, \ldots, P_{n_{s_{j}}}$ when we desingularize $\bar{X}_{\mathrm{a}}$. Without loss of generality, we shall assume that $\lambda_{j}=0$. The polynomial $P(x, y, z)$ is homogeneous in $\mathbb{P}^{2}(d)$ of degree $r d$ :

$$
P(x, y, z)=\operatorname{det}\left(z^{d} A(x / z)-y \mathrm{Id}_{r}\right) .
$$

Write $P(1, y, z)$ as the sum of homogeneous polynomials in ascending degrees

$$
P(1, y, z)=P_{s_{j}}(y, z)+P_{s_{j}+1}(y, z)+\cdots+P_{r d}(y, z) \text {. }
$$


Precisely

$$
\begin{aligned}
& P_{s_{j}}(y, z)=\prod_{i \neq j} \lambda_{i}^{s_{i}} \operatorname{det}\left(z u_{d-1}^{j}-y \operatorname{Id}_{s_{j}}\right), \\
& P_{s_{j}}(y, z)=\prod_{i \neq j} \lambda_{i}^{s_{i}} \prod_{i=1}^{s_{j}}\left(z \alpha_{i}-y\right) .
\end{aligned}
$$

Let $\phi_{n_{i}}$ be the eigenvector of $A(x)$ near the points $P_{n_{i}}$ for $i=1,2, \ldots, s_{j}$. We have

$$
y=\alpha_{n_{i}} z+\cdots .
$$

We put $\phi_{n_{i}}=e_{n_{i}}+v_{n_{i}} z+\cdots$, and get

$$
\left(A_{d}+A_{d-1} z+\cdots\right)\left(e_{n_{i}}+v_{n_{i}} z+\cdots\right)=\left(\alpha_{n_{i}} z+\cdots\right)\left(e_{n_{i}}+v_{n_{i}} z+\cdots\right),
$$

or, componentwise,

$$
\begin{gathered}
A_{d} e_{n_{i}}=0 \\
A_{d} v_{n_{i}}+A_{d-1} e_{n_{i}}=\alpha_{n_{i}} e_{n_{i}} .
\end{gathered}
$$

We get that $e_{n_{i}}$ corresponds to the eigenvector of $\left(\delta_{k}^{n_{i}}\right)_{1 \leqslant k \leqslant r}$.

Notice the vectors $f\left(P_{i}\right)=\left(f_{1}\left(P_{i}\right), \ldots, f_{r}\left(P_{i}\right)\right)$ are fixed and depend on the spectral curve $\bar{X}_{\mathrm{a}}$ at infinity. Let $c \in \mathbb{C}^{r}$ and consider the hyperplane $H_{c}=\left\{v \in \mathbb{C}^{r}:\langle c, v\rangle=\right.$ $\left.c_{1} v_{1}+\cdots+c_{r} v_{r}=0\right\}$. To normalize the eigenvector $f$, we choose $c \in \mathbb{C}^{r}$ such that $f\left(P_{i}\right) \notin H_{c}$ (in our case, we can choose $c=(1, \ldots, 1)$ ), and the normalization of $f$ is given by $\langle c, f\rangle \equiv 1, f$ has a pole at $P_{i}$ if and only if the line determined by $f\left(P_{i}\right)$ is contained in the plane $c_{1} f_{1}+c_{2} f_{2}+\cdots+c_{r} f_{r}=0$, so with the above normalization $P_{i}$ is not a pole for $f$.

If $D$ is the pole divisor of the normalized eigenvector $f$, then denote by $L=L_{D}$ the line bundle and by $\mathcal{L}=\mathcal{L}_{D}$ the corresponding sheaf of sections.

\section{The dimension of $H^{0}(X, \mathcal{L}(D))$}

To calculate $\operatorname{dim} H^{0}(X, \mathcal{L}(D))$ we follow [18]. Let $(x)_{\infty}=\sum_{i=1}^{r} P_{i}$ and introduce $X_{0}=$ $X-\left\{P_{1}, P_{2}, \ldots, P_{r}\right\}$ and let $R_{0}$ be the algebra of regular functions in $X_{0}$. Clearly $R_{0}=$ $\mathbb{C}[x, y]$. Put $R=\mathbb{C}[x]$. Let $V$ denote the subspace of $H^{0}\left(X_{0}, \mathcal{L}(D)\right)$ generated by linear coordinates in $\mathbb{C}^{r}$. Now we prove that the natural mapping $r: V \otimes R \rightarrow H^{0}\left(X_{0}, \mathcal{L}(D)\right)$ is surjective. $r(V \otimes R)$ is an $R_{0}$-module if $f=\left(f_{1}, \ldots, f_{r}\right)$ is the standard basis in $V$, then $y f=A(x) f$ so that $y f_{i} \in r(V \otimes R)$. Suppose that $r(V \otimes R)$ is a proper $R_{0}$-submodule in $H^{0}\left(X_{0}, \mathcal{L}(D)\right)$. Then there is a maximal ideal $I_{\mathrm{m}}$ in $R_{0}$ such that

$$
r(V \otimes R) \subset I_{\mathrm{m}} H^{0}\left(X_{0}, \mathcal{L}(D)\right)
$$

A maximal ideal $I_{\mathrm{m}}$ in $R_{0}$ is naturally associated with a point $p \in X_{0}$ :

$$
I_{\mathrm{m}}=\left\{g \in R_{0}, g(p)=0\right\} .
$$


All functions in $r(V \otimes R)$ would vanish at $p$, while $1=\sum_{i=1}^{r} f_{i}$ contradicts this fact. Hence, any function of $f \in L(D)$ is an element in $r(V \otimes R)$ :

$$
f=\sum_{i=1}^{r} b_{i} f_{i}+x\left(\sum_{i=1}^{r} b_{i}^{1} f_{i}\right)+\cdots+x^{\alpha}\left(\sum_{i=1}^{r} b_{i}^{\alpha} f_{i}\right) .
$$

Moreover,

$$
f x^{-\alpha}\left(P_{j}\right)=\sum_{i=1}^{r} b_{i} f_{i} x^{-\alpha}\left(P_{j}\right)+x^{1-\alpha}\left(P_{j}\right)\left(\sum_{i=1}^{r} b_{i}^{1} f_{i}\left(P_{j}\right)\right)+\cdots+\left(\sum_{i=1}^{r} b_{i}^{\alpha} f_{i}\left(P_{j}\right)\right) .
$$

We get

$$
\sum_{i=1}^{r} b_{i}^{\alpha} f_{i}\left(P_{j}\right)=0, \quad 1 \leqslant j \leqslant r .
$$

The $r$ eigenvectors $\left(f_{i}\left(P_{j}\right)\right)_{1 \leqslant i \leqslant r}$ are independent because the matrix $J$ is diagonalizable, and $\operatorname{det}\left(f_{i}\left(P_{j}\right)\right)_{1 \leqslant i, j \leqslant r} \neq 0$, implying $b_{i}^{\alpha}=0$ for $1 \leqslant i \leqslant r$. In the same way, $b_{i}^{j}=0$ for $1 \leqslant i \leqslant r$ and $1 \leqslant j \leqslant \alpha$, proving that

$$
f=\sum_{i=1}^{r} b_{i} f_{i}
$$

Therefore $\operatorname{dim} H^{0}(X, \mathcal{L}(D))=r$.

\section{Calculation of $\operatorname{deg}(D)$}

We consider $E=D+n \sum_{i=1}^{r} P_{i}$ with $n$ large enough. Clearly $\operatorname{dim} L(E) \geqslant r(n+1)$, since every function $x^{i} f_{j}$ for $0 \leqslant i \leqslant n$ and $1 \leqslant j \leqslant r$ belongs to that space. But every function $f \in L(E)$ belongs to $r(V \otimes R)$. So that

$$
f=\sum_{i=1}^{r} b_{i} f_{i}+x\left(\sum_{i=1}^{r} b_{i}^{1} f_{i}\right)+\cdots+x^{\alpha}\left(\sum_{i=1}^{r} b_{i}^{\alpha} f_{i}\right) .
$$

Using the same process, it is easy to show that $\alpha \leqslant n$. Therefore, the dimension of $L(E)$ is $r(n+1)$. Using the Riemann-Roch Theorem on $X$ we get

$$
r n+\operatorname{deg}(D)-p_{\mathrm{g}}+1=\operatorname{dim} L(E)=r(n+1) .
$$

So that

$$
\operatorname{deg}(D)=p_{\mathrm{g}}+r-1
$$

\section{Statement of the main result}

Firstly, we state the next proposition. 
Proposition 7.1. We define $\Theta$ as the canonical Riemann theta divisor of $J^{p_{a}}(X)$ formed by special line bundles $L_{D}$ of degree $p_{\mathrm{a}}$, i.e. $\operatorname{dim} H^{1}(X, \mathcal{L}(D)) \neq 0$. If $L_{D} \in$ $\mathrm{Pic}^{p_{\mathrm{a}}}(X)-\Theta$, then the sheaf $\pi_{*} \mathcal{L}(D)$ ( $\pi$ is the projection $X \rightarrow \mathbb{P}^{1}$ induced by $[x, y, z] \mapsto$ $[x, z])$ is a trivial $\mathcal{O}_{\mathbb{P} 1}$-module of rank $r$ :

$$
\pi_{*} \mathcal{L}(D)=\mathcal{O}_{P^{1}} \oplus \mathcal{O}_{\mathbb{P} 1} \oplus \cdots \oplus \mathcal{O}_{P^{1}}
$$

Then we have a bi-holomorphic map

$\left\{\right.$ a matrix $A(x) \in M_{P}$ up to conjugation by a matrix in $\left.\mathbb{P} G L_{r}(\mathbb{C})\right\}$

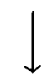

$\left\{\right.$ an isomorphism class of a line bundle $\left.L \in \mathrm{Pic}^{p_{\mathbf{a}}}(X)-\Theta\right\}$.

Proof. The demonstration of [3] works with little modifications. Let $L$ be a line bundle on $X$ of degree $\operatorname{deg}(L)=p_{\mathrm{a}}$ and $\operatorname{dim} H^{0}(X, \mathcal{L})=r$. By the Riemann-Roch Theorem, ${ }^{*}$ $\chi(\mathcal{L})=\operatorname{deg}(L)-p_{\mathrm{g}}+1=r$. Moreover

$$
\begin{gathered}
\operatorname{dim} H^{0}(X, \mathcal{L})=\operatorname{dim} H^{0}\left(\mathbb{P}^{1}, \pi_{*} \mathcal{L}\right) \\
\chi(\mathcal{L})=\chi\left(\pi_{*} \mathcal{L}\right)=\operatorname{deg}\left(\pi_{*} \mathcal{L}\right)+\left(1-p_{\mathrm{g}}\left(\mathbb{P}^{1}\right)\right) \operatorname{rank}\left(\pi_{*} \mathcal{L}\right)=\operatorname{deg}\left(\pi_{*} \mathcal{L}\right)+r
\end{gathered}
$$

Then we obtain that $\pi_{*} \mathcal{L}$ is a locally trivial $\mathcal{O}_{\mathbb{P}^{1}}$-module of rank $r$, degree 0 and it has $r$ holomorphic sections, so

$$
\pi_{*} \mathcal{L}=\underbrace{\mathcal{O}_{\mathbb{P}^{1}} \oplus \mathcal{O}_{\mathbb{P}^{1}} \oplus \cdots \oplus \mathcal{O}_{\mathbb{P}^{1}}}_{r}
$$

The invertible sheaf $\mathcal{L}$ on $X$ can be equivalently described as a locally trivial $\mathcal{O}_{\mathbb{P}^{1}}$-module $\pi_{*} \mathcal{L}$ equipped with an additional structure of a $\pi_{*} \mathcal{O}$-module, or, equivalently, a homomorphism of algebras $a: \pi_{*} \mathcal{O} \rightarrow \operatorname{End}\left(\pi_{*} \mathcal{L}\right)$. To describe the homomorphism $a$ amounts to giving a linear map

$$
A: \pi_{*} \mathcal{L} \rightarrow \pi_{*} \mathcal{L}(d)
$$

that is to say, a polynomial $r \times r$ matrix $A(x)$ of degree $d$. Clearly, $A(x)$ satisfies $P(x, A(x))=0$ and as $P(x, y)$ is irreducible over $\mathbb{C}(x)$, then by the Cayley-Hamilton Theorem the spectral polynomial of $A(x)$ is $P(x, y)$. Note that the matrix $A(x)$ is determined only modulo an automorphism of $\pi_{*} \mathcal{L}$, and hence up to conjugation by a matrix $R \in G L_{r}(\mathbb{C})$.

Denote

$$
\mathbb{P} G L_{r}(\mathbb{C} ; \mathcal{F})=\left\{R \in \mathbb{P} G L_{r}(\mathbb{C}): R \text { is a diagonal matrix }\right\}
$$

* $\chi(\mathcal{L})$ is the Euler-Poincaré characteristic defined for a line bundle $L$ on $X$ by

$$
\chi(\mathcal{L})=\operatorname{dim} H^{0}(X, \mathcal{L})-\operatorname{dim} H^{1}(X, \mathcal{L}) .
$$


The group $\mathbb{P} G L_{r}(\mathbb{C} ; \mathcal{F})$ acts freely and properly on the smooth manifold $M_{P}^{\mathcal{F}}$ by conjugation. We consider the following commutative diagram

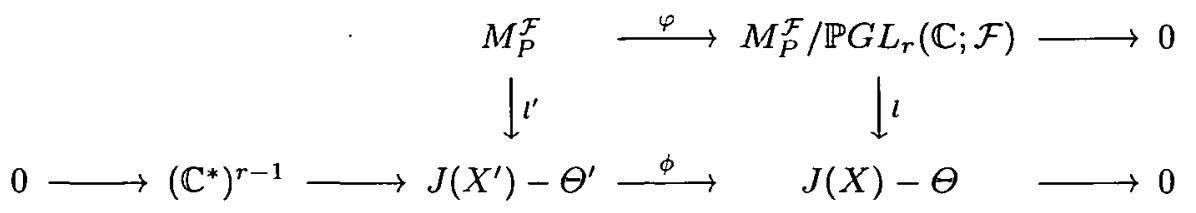

where $\Theta^{\prime}=\phi^{-1}(\Theta)$. We are ready to state the following theorem.

Theorem 7.2. Denote by $\Theta^{\prime}=\phi^{-1}(\theta)$ the theta divisor formed by line bundles $L^{\prime} \in$ $\operatorname{Pic}^{p_{\mathrm{a}}}\left(X^{\prime}\right)$ such that $\operatorname{dim} H^{1}\left(X^{\prime}, L^{\prime}\right) \neq 0$. The variety $M_{P}^{\mathcal{F}}$ is smooth and bi-holomorphic to $J\left(X^{\prime}\right)-\Theta^{\prime}$.

Proof. Let $\mathrm{Pic}^{p_{\mathrm{a}}}\left(X^{\prime}\right)$ be the shifted Picard group $\mathrm{Pic}^{0}\left(X^{\prime}\right)$ of degree $p_{\mathrm{a}}$ line bundles on $X^{\prime}$. It is isomorphic to the Jacobian variety $J\left(X^{\prime}\right)$, and $J\left(X^{\prime}\right)-\Theta^{\prime}$ is the subset of line bundles $L^{\prime} \in \mathrm{Pic}^{p_{\mathrm{a}}}\left(X^{\prime}\right)$ with one non-zero holomorphic section. We get a mapping

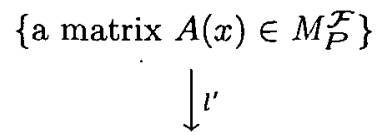

\{an isomorphism class of a line bundle $\left.L \in \mathrm{Pic}^{p_{\mathrm{a}}}\left(X^{\prime}\right)-\Theta^{\prime}\right\}$.

In fact it is an isomorphism, to show that we follow [8] and prove the fibres $\varphi^{-1}(b)$ and $\phi^{-1} \circ l(b)$ have the same dimension and $l^{\prime}$ is an injective morphism of algebraic groups. The dimension of the fibres is $r-1$. Now check that $l^{\prime}$ is injective. Let $L^{\prime}$ be a line bundle on $X^{\prime}$ of degree $\operatorname{deg}\left(L^{\prime}\right)=p_{\mathrm{a}}$ and $\operatorname{dim} H^{0}\left(X^{\prime}, \mathcal{L}^{\prime}\right)=1$. By the Riemann-Roch Theorem $\chi\left(\mathcal{L}^{\prime}\right)=\operatorname{deg}\left(L^{\prime}\right)-p_{\mathrm{a}}+1=1$. Moreover,

$$
\begin{gathered}
\operatorname{dim} H^{0}\left(X^{\prime}, \mathcal{L}^{\prime}\right)=\operatorname{dim} H^{0}\left(\mathbb{P}^{1}, \pi_{*} \mathcal{L}^{\prime}\right) \\
\chi\left(\mathcal{L}^{\prime}\right)=\chi\left(\pi_{*} \mathcal{L}^{\prime}\right)=\operatorname{deg}\left(\pi_{*} \mathcal{L}^{\prime}\right)+\left(1-p_{\mathrm{g}}\left(\mathbb{P}^{1}\right)\right) \operatorname{rank}\left(\pi_{*} \mathcal{L}^{\prime}\right)=\operatorname{deg}\left(\pi_{*} \mathcal{L}^{\prime}\right)+r .
\end{gathered}
$$

Then we obtain that $\pi_{*} \mathcal{L}^{\prime}$ is a locally trivial $\mathcal{O}_{\mathbb{P}^{1}}$-module of rank $r$, degree $1-r$ and has one holomorphic section, so

$$
\pi_{*} \mathcal{L}^{\prime}=\underbrace{\mathcal{O}_{P^{1}} \oplus \mathcal{O}_{\mathbb{P}^{1}}(-1) \oplus \cdots \oplus \mathcal{O}_{\mathbb{P}^{1}}(-1)}_{r}
$$

The invertible sheaf $\mathcal{L}^{\prime}$ on $X^{\prime}$ can be equivalently described as a locally trivial $\mathcal{O}_{\geq 1}$-module $\pi_{*} \mathcal{L}^{\prime}$ equipped with an additional structure of a $\pi_{*} \mathcal{O}^{\prime}$-module, therefore a homomorphism of algebras $a: \pi_{*} \mathcal{O}^{\prime} \rightarrow \operatorname{End}\left(\pi_{*} \mathcal{L}^{\prime}\right)$. To describe the homomorphism $a$ we shall give a linear map

$$
A: \pi_{*} \mathcal{L}^{\prime} \rightarrow \pi_{*} \mathcal{L}^{\prime}(d),
$$

that is to say, a polynomial $r \times r$ matrix $A(x)$ of degree $d$. Clearly, $A(x)$ satisfies $P(x, A(x))=0$ and as $P(x, y)$ is irreducible over $\mathbb{C}(x)$, then by the Cayley-Hamilton 
Theorem the spectral polynomial of $A(x)$ is $P(x, y)$. Note that the matrix $A(x)$ is determined only modulo an automorphism of $\pi_{*} \mathcal{L}^{\prime}$. As the vector $1=\sum f_{i} \in H^{0}\left(\mathbb{P}^{1}, \pi_{*} \mathcal{L}^{\prime}\right)$ in the base $f_{1}, f_{2}, \ldots, f_{r}$, is the vector $(1,1, \ldots, 1)$, then $A(x)$ is determined up to conjugation by a constant matrix $R$ with eigenvector $(1,1, \ldots, 1)$. Thus if $A^{\prime}(x)$ is another matrix in $M_{P}^{\mathcal{F}}$ which defines the same eigenvector sheaf $\mathcal{L}^{\prime}$, then $A^{\prime}(x)=R A(x) R^{-1}$. As $R$ commutes with the leading term $J, R_{E_{d-1}}$ commutes with $u_{d-1}$ and $(1,1, \ldots, 1)$ is an eigenvector of $R$, then $R=\operatorname{Id} \in \mathbb{P} G L_{r}(\mathbb{C})$.

Finally it remains to show that $M_{P}^{\mathcal{F}}$ is smooth. We choose $a \in \mathbb{P}^{1}, a \neq[0: 1]$ generic (i.e. such that $A(a)$ has a simple spectrum), the vector fields

$$
\frac{\mathrm{d}}{\mathrm{d} t} A(x)=\left[\frac{A^{k}(a)}{x-a}, A(x)\right], \quad k \in \mathbb{N},
$$

are tangent to the fibre $\varphi^{-1}(b)$. It is shown in [14] that these vector fields induce translation invariant vector fields on $J\left(X^{\prime}\right)$ (although the results are formulated only on $J(X)$ ). The direction of these vector fields is moreover computed (formula (8.5) on p. 177 of [14]). These formulae imply that these vector fields span, for $a$ generic, the tangent space to the generalized Jacobian $J\left(X^{\prime}\right)$.

\section{Examples}

\section{Example 1: a harmonic oscillator with two degrees of freedom}

Consider the simplest case, where $A(x)$ is written in the base $\left\{e_{1}, e_{2}, e_{3}\right\}$ :

$$
A(x)=A_{1} x+A_{0}=\left(\begin{array}{cc|c}
0 & 0 & 0 \\
0 & 0 & 0 \\
\hline 0 & 0 & a_{0}
\end{array}\right) x+\left(\begin{array}{cc|c}
a_{1} & 0 & y_{1} \\
0 & a_{2} & y_{2} \\
\hline x_{1} & x_{2} & a_{3}
\end{array}\right) .
$$

The spectral polynomial $P(x, y)=\operatorname{det}\left(A(x)-y \operatorname{Id}_{r}\right)$ is

$$
P(x, y)=h_{1}+h_{2} y+h_{3} y^{2}-y^{3}+x\left(h_{4}+h_{5} y+a_{0} y^{2}\right) .
$$

When $a_{1}=a_{2}$, the polynomial $P(x, y)$ is not irreducible, so consider the case $a_{1} \neq a_{2}$. Let

$$
X_{\mathrm{a}}=\{(x, y): P(x, y)=0\} \subset \mathbb{P}^{2} .
$$

Let $\bar{X}_{\mathrm{a}}$ denote the closure of the affine curve $X_{\mathrm{a}}$ in the total space of the line bundle $\mathcal{O}_{\mathbb{P}^{1}}(1)$. We choose $a_{0} \in \mathbb{C}^{*}$ and $\left(h_{1}, h_{2}, h_{3}, h_{4}, h_{5}\right) \in \mathbb{C}^{5}$ such that $\bar{X}_{\mathrm{a}}-Q$ is smooth, and then at $Q(x(Q)=\infty$ and $y(Q)=0)$, the curve $\bar{X}_{\mathrm{a}}$ is locally a normal crossing of two branches. Denote by $X$ the smooth compactification of $\bar{X}_{\mathrm{a}}$. The double point $Q \in \bar{X}_{\mathrm{a}}$ gives two smooth points $P_{1}, P_{2} \in X$ on $\bar{X}_{\mathrm{a}}$. Therefore, $X$ is topologically a sphere. Let $P_{3}=\left(x=\infty, y=a_{0}\right)$, the modulus is $\mathfrak{m}=P_{1}+P_{2}+P_{3}$ and call $X^{\prime}=X_{\mathfrak{m}}$,

$$
\begin{aligned}
P(x, y, z)= & a_{0} a_{1} a_{2} x z^{2}-a_{0}\left(a_{1}+a_{2}\right) x y z+a_{0} x y^{2}-y^{3} \\
& +\left(a_{1}+a_{2}+a_{3}\right) y^{2} z+\left(x_{1} y_{1}+x_{2} y_{2}-a_{1} a_{2}-a_{1} a_{3}-a_{2} a_{3}\right) y z^{2} \\
& +\left(a_{1} a_{2} a_{3}-a_{2} x_{1} y_{1}-a_{1} x_{2} y_{2}\right) z^{3} .
\end{aligned}
$$


The data of $\bar{X}_{\mathrm{a}}$ at infinity is

$$
\mathcal{F}=\left\{\left(\left\{e_{1}, e_{2}, e_{3}\right\}, A_{1}\right),\left(\left\{e_{1}, e_{2}\right\},\left(\begin{array}{cc}
a_{1} & 0 \\
0 & a_{2}
\end{array}\right)\right)\right\}
$$

The variety $M_{P}^{\mathcal{F}}$ is defined by

$$
\left.M_{P}^{\mathcal{F}}=\left\{\begin{array}{l}
a_{1} \\
a_{2} \\
a_{3} \\
x_{1} \\
x_{2} \\
y_{1} \\
y_{2}
\end{array}\right) \in \mathbb{C}^{7}: \begin{array}{r}
a_{1} a_{2} a_{3}-a_{2} x_{1} y_{1}-a_{1} x_{2} y_{2}=h_{1} \\
y_{1} x_{1}+y_{2} x_{2}-a_{1} a_{2}-a_{1} a_{3}-a_{2} a_{3}=h_{2} \\
a_{1}+a_{2}+a_{3}=h_{3} \\
a_{0} a_{1} a_{2}=h_{4} \\
-a_{0}\left(a_{1}+a_{2}\right)=h_{5}
\end{array}\right\}
$$

The Hamiltonian system on $M_{P}^{\mathcal{F}}$ is $\dot{A}_{0}=\left[A_{0}, A_{1}\right]$, or, in others words,

$$
\begin{aligned}
& \dot{y_{1}}=a_{0} y_{1}, \\
& \dot{y_{2}}=a_{0} y_{2}, \\
& \dot{x_{1}}=-a_{0} x_{1}, \\
& \dot{x_{2}}=-a_{0} x_{2}, \\
& \dot{a_{1}}=0, \\
& \dot{a_{2}}=0, \\
& \dot{a_{3}}=0 .
\end{aligned}
$$

The Hamiltonian function is

$$
H(A(x))=\frac{1}{2} \operatorname{Res}_{x=0} \operatorname{tr}\left(A^{2}(x)\right) \frac{\mathrm{d} x}{x}
$$

so

$$
H(A(x))=y_{1} x_{1}+y_{2} x_{2} .
$$

The Poisson bracket $\{\cdot, \cdot\}_{1}$ is given by

$$
\left\{y_{1}, x_{1}\right\}_{1}=-a_{0}, \quad\left\{y_{2}, x_{2}\right\}_{1}=-a_{0}, \quad\left\{y_{i}, x_{j}\right\}_{1}=\left\{x_{i}, y_{j}\right\}_{1}=0, \quad \text { for } i \neq j
$$

The functions $a, a_{1}, a_{2}$ and $a_{3}$ are Casimir functions for this structure.

Remark 8.1. We can take

$$
H_{k}(A(x))=\frac{1}{2} R e s_{x=0} \operatorname{tr}\left(A^{2}(x)\right) \frac{\mathrm{d} x}{x^{k}}, \quad k=2,3,
$$

as the Hamiltonian function for the above system. Following the scheme described by Reyman [14], we get other compatible Poisson structures. For instance,

$$
H_{2}=a_{0} a_{3}
$$


with the Poisson bracket

\begin{tabular}{c|cccccccc}
$\{\cdot, \cdot\}_{2}$ & $a_{0}$ & $a_{1}$ & $a_{2}$ & $a_{3}$ & $y_{1}$ & $y_{2}$ & $x_{1}$ & $x_{2}$ \\
\hline$a_{0}$ & 0 & 0 & 0 & 0 & 0 & 0 & 0 & 0 \\
$a_{1}$ & 0 & 0 & 0 & 0 & $y_{1}$ & 0 & $-x_{1}$ & 0 \\
$a_{2}$ & 0 & 0 & 0 & 0 & 0 & $y_{2}$ & 0 & $-x_{2}$ \\
$a_{3}$ & 0 & 0 & 0 & 0 & $-y_{1}$ & $-y_{2}$ & $x_{1}$ & $x_{2}$ \\
$y_{1}$ & 0 & $-y_{1}$ & 0 & $y_{1}$ & 0 & 0 & $a_{1}-a_{3}$ & 0 \\
$y_{2}$ & 0 & 0 & $-y_{2}$ & $y_{2}$ & 0 & 0 & 0 & $a_{2}-a_{3}$ \\
$x_{1}$ & 0 & $x_{1}$ & 0 & $-x_{1}$ & $a_{3}-a_{1}$ & 0 & 0 & 0 \\
$x_{2}$ & 0 & 0 & $x_{2}$ & $-x_{2}$ & 0 & $a_{3}-a_{2}$ & 0 & 0
\end{tabular}

or again we may consider

$$
H_{3}=\frac{1}{2} a_{0}^{2}
$$

with the following Poisson bracket

\begin{tabular}{c|cccccccc}
$\{\cdot, \cdot\}_{3}$ & $a_{0}$ & $a_{1}$ & $a_{2}$ & $a_{3}$ & $y_{1}$ & $y_{2}$ & $x_{1}$ & $x_{2}$ \\
\hline$a_{0}$ & 0 & 0 & 0 & 0 & $-y_{1}$ & $-y_{2}$ & $x_{1}$ & $x_{2}$ \\
$a_{1}$ & 0 & 0 & 0 & 0 & 0 & 0 & 0 & 0 \\
$a_{2}$ & 0 & 0 & 0 & 0 & 0 & 0 & 0 & 0 \\
$a_{3}$ & 0 & 0 & 0 & 0 & 0 & 0 & 0 & 0 \\
$y_{1}$ & $y_{1}$ & 0 & 0 & 0 & 0 & 0 & 0 & 0 \\
$y_{2}$ & $y_{2}$ & 0 & 0 & 0 & 0 & 0 & 0 & 0 \\
$x_{1}$ & $-x_{1}$ & 0 & 0 & 0 & 0 & 0 & 0 & 0 \\
$x_{2}$ & $-x_{2}$ & 0 & 0 & 0 & 0 & 0 & 0 & 0
\end{tabular}

The components of the eigenvector of $A(x)$ are given by

$$
f_{1}=y_{1}\left(a_{2}-y\right), \quad f_{2}=\left(a_{1}-y\right) y_{2}, f_{3}=\left(a_{1}-y\right)\left(a_{2}-y\right) .
$$

We normalize these functions by dividing by $g=f_{1}+f_{2}+f_{3}=y^{2}-\left(a_{1}+a_{2}+y_{1}+\right.$ $\left.y_{2}\right) y+a_{1} a_{2}+a_{1} y_{2}+y_{1} a_{2}$. This polynomial $g$ has two roots denoted $y^{0}$ and $y^{1}$. At each value of $y$, there is one value of $x$, we call $x^{0}$ the value of $x$ for $y^{0}$ and $x^{1}$ for $y^{1}$. Let $Q_{0}=\left(x^{0}, y^{0}\right)$ and $Q_{1}=\left(x^{1}, y^{1}\right)$. We get

$$
\left(f_{k} / g\right) \geqslant-Q_{0}-Q_{1}, \quad k=1,2,3 .
$$

We put $D=Q_{0}+Q_{1}, \operatorname{deg}(D)=2$. Using Theorem 7.2 , we get that the variety $M_{P}^{\mathcal{F}}$ is an open given by $J^{2}\left(X^{\prime}\right)-\Theta^{\prime}$. $^{*}$

* Let $J^{p_{\mathbf{a}}}\left(X^{\prime}\right)=\operatorname{Pic}^{p_{a}}\left(X^{\prime}\right)$ be the variety (isomorphic to the generalized Jacobian $J\left(X^{\prime}\right)$ ) formed by line bundles of degree $p_{\mathrm{a}}$ (the arithmetic genus of the singularized curve $X^{\prime}$ ) over $X^{\prime}$. 


\section{Example 2: integrable perturbation of the harmonic oscillator with five degrees of freedom}

Now consider the Hamiltonian function

$$
\begin{aligned}
H=\left(a_{1}+a_{2}\right) p_{1}^{2}+\left(a_{1}+b_{2}\right) p_{2}^{2}+\left(b_{1}+a_{2}\right) p_{3}^{2}+\left(b_{1}+b_{2}\right) p_{4}^{2}+\left(a_{2}+b_{2}\right) p_{5}^{2} \\
-\left(a_{1}+a_{2}\right) q_{1}^{2}-\left(a_{1}+b_{2}\right) q_{2}^{2}-\left(b_{1}+a_{2}\right) q_{3}^{2}-\left(b_{1}+b_{2}\right) q_{4}^{2}-\left(a_{2}+b_{2}\right) q_{5}^{2}
\end{aligned}
$$

defined on the co-tangent bundle $T^{*} \mathbb{C}^{5}$ of $\mathbb{C}^{5}$, equipped with a canonical symplectic form $\omega=\mathrm{d} p_{1} \wedge \mathrm{d} q_{1}+\mathrm{d} p_{2} \wedge \mathrm{d} q_{2}+\mathrm{d} p_{3} \wedge \mathrm{d} q_{3}+\mathrm{d} p_{4} \wedge \mathrm{d} q_{4}+\mathrm{d} p_{5} \wedge \mathrm{d} q_{5}$. Under a simple transformation

$$
\begin{array}{ll}
p_{i}=\frac{1}{\sqrt{ } 2}\left(x_{i}+y_{i}\right), & q_{i}=\frac{1}{\sqrt{ } 2}\left(-x_{i}+y_{i}\right), \quad i=1, \ldots, 4, \\
p_{5}=\frac{1}{2}\left(x_{5}+y_{5}\right), & q_{5}=\frac{1}{2}\left(-x_{5}+y_{5}\right),
\end{array}
$$

the Hamiltonian function becomes

$$
H=\left(a_{1}+a_{2}\right) x_{1} y_{1}+\left(a_{1}+b_{2}\right) x_{2} y_{2}+\left(b_{1}+a_{2}\right) x_{3} y_{3}+\left(b_{1}+b_{2}\right) x_{4} y_{4}+\left(a_{2}+b_{2}\right) x_{5} y_{5}
$$

and the symplectic structure becomes:

\begin{tabular}{c|cccccccccc}
$\{\cdot, \cdot\}_{4}$ & $x_{1}$ & $x_{2}$ & $x_{3}$ & $x_{4}$ & $x_{5}$ & $y_{1}$ & $y_{2}$ & $y_{3}$ & $y_{4}$ & $y_{5}$ \\
\hline$x_{1}$ & 0 & 0 & 0 & 0 & 0 & 1 & 0 & 0 & 0 & 0 \\
$x_{2}$ & 0 & 0 & 0 & 0 & 0 & 0 & 1 & 0 & 0 & 0 \\
$x_{3}$ & 0 & 0 & 0 & 0 & 0 & 0 & 0 & 1 & 0 & 0 \\
$x_{4}$ & 0 & 0 & 0 & 0 & 0 & 0 & 0 & 0 & 1 & 0 \\
$x_{5}$ & 0 & 0 & 0 & 0 & 0 & 0 & 0 & 0 & 0 & 2 \\
$y_{1}$ & -1 & 0 & 0 & 0 & 0 & 0 & 0 & 0 & 0 & 0 \\
$y_{2}$ & 0 & -1 & 0 & 0 & 0 & 0 & 0 & 0 & 0 & 0 \\
$y_{3}$ & 0 & 0 & -1 & 0 & 0 & 0 & 0 & 0 & 0 & 0 \\
$y_{4}$ & 0 & 0 & 0 & -1 & 0 & 0 & 0 & 0 & 0 & 0 \\
$y_{5}$ & 0 & 0 & 0 & 0 & -2 & 0 & 0 & 0 & 0 & 0
\end{tabular}

We perturb $H$, adding terms of order three

$$
\begin{aligned}
H^{\prime}=\left(a_{1}+a_{2}\right) x_{1} y_{1}+\left(a_{1}+b_{2}\right) & x_{2} y_{2}+\left(b_{1}+a_{2}\right) x_{3} y_{3}+\left(b_{1}+b_{2}\right) x_{4} y_{4} \\
& +\left(a_{2}+b_{2}\right) x_{5} y_{5}+\left(x_{1} x_{2}+x_{3} x_{4}\right) y_{5}+\left(y_{1} y_{2}+y_{3} y_{4}\right) x_{5}
\end{aligned}
$$

Proposition 8.2. The system $\left(H^{\prime},\{\cdot, \cdot\}_{4}, \mathbb{R}^{10}\right)$ is algebraically completely integrable.

Note in the base $\left\{e_{1}, e_{2}, e_{3}, e_{4}\right\}$

$$
A(x)=A_{1} x+A_{0}=\left(\begin{array}{cc|cc}
0 & 0 & 0 & 0 \\
0 & 0 & 0 & 0 \\
\hline 0 & 0 & 1 & 0 \\
0 & 0 & 0 & -1
\end{array}\right) x+\left(\begin{array}{cc|cc}
a_{1} & 0 & y_{1} & x_{2} \\
0 & b_{1} & y_{3} & x_{4} \\
\hline x_{1} & x_{3} & a_{2} & x_{5} \\
y_{2} & y_{4} & y_{5} & b_{2}
\end{array}\right) .
$$


The Hamiltonian system is $\dot{A}_{0}=\left[A_{1}, A_{0}^{2}\right]$

$$
\begin{array}{ll}
\dot{x_{1}}=\left(a_{1}+a_{2}\right) x_{1}+y_{2} x_{5}, & \dot{y_{1}}=-\left(a_{1}+a_{2}\right) y_{1}-x_{2} y_{5}, \\
\dot{x_{2}}=\left(a_{1}+b_{2}\right) x_{2}+y_{1} x_{5}, & \dot{y_{2}}=-\left(a_{1}+b_{2}\right) y_{2}-x_{1} y_{5}, \\
\dot{x_{3}}=\left(a_{2}+b_{1}\right) x_{3}+y_{4} x_{5}, & \dot{y_{3}}=-\left(a_{2}+b_{1}\right) y_{3}-x_{4} y_{5}, \\
\dot{x_{4}}=\left(b_{1}+b_{2}\right) x_{4}+y_{3} x_{5}, & \dot{y_{4}}=-\left(b_{1}+b_{2}\right) y_{4}-x_{3} y_{5}, \\
\dot{x_{5}}=2\left(a_{2}+b_{2}\right) x_{5}+2\left(x_{1} x_{2}+x_{3} x_{4}\right), & \dot{y_{5}}=-2\left(a_{2}+b_{2}\right) y_{5}-2\left(y_{1} y_{2}+y_{3} y_{4}\right), \\
\dot{a_{2}}=0, & \dot{b_{2}}=0 .
\end{array}
$$

The corresponding Hamiltonian function is

$$
\begin{aligned}
H_{1}(A(x))= & \frac{1}{3} \operatorname{Res}_{x=0} \operatorname{tr}\left(A^{3}(x)\right) \frac{\mathrm{d} x}{x} \\
= & \left(a_{1}+a_{2}\right) x_{1} y_{1}+\left(a_{1}+b_{2}\right) x_{2} y_{2}+\left(b_{1}+a_{2}\right) x_{3} y_{3}+\left(b_{1}+b_{2}\right) x_{4} y_{4} \\
& \quad+\left(a_{2}+b_{2}\right) x_{5} y_{5}+\left(x_{1} x_{2}+x_{3} x_{4}\right) y_{5}+\left(y_{1} y_{2}+y_{3} y_{4}\right) x_{5}
\end{aligned}
$$

with the Poisson bracket of (8.1).

Remark 8.3. As in the previous example, this system possesses another Poisson structure compatible with the first one. We find the same system with $H_{2}=x_{1} y_{1}$ $x_{2} y_{2}+x_{3} y_{3}-x_{4} y_{4}+a_{2}^{2}-b_{2}^{2}$ and the Poisson structure $\{\cdot, \cdot\}_{5}$ (see Table 1 ).

Fix the polynomial

$$
\begin{aligned}
P(x, y)= & \operatorname{det}\left(A(x)-y \operatorname{Id}_{r}\right) \\
= & h_{1}+h_{2} x+h_{3} y-a_{1} b_{1} x^{2}+h_{4} x y+h_{5} y^{2}+\left(a_{1}+b_{1}\right) x^{2} y \\
& \quad+\left(b_{2}-a_{2}\right) x y^{2}-\left(a_{1}+b_{1}+a_{2}+b_{2}\right) y^{3}-x^{2} y^{2}+y^{4}
\end{aligned}
$$

where $a_{1} \neq b_{1}$ and $a_{2}, b_{2}, h_{i} \in \mathbb{C}$. Note that

$$
X_{\mathrm{a}}=\left\{(x, y) \in \mathbb{C}^{2}: P(x, y)=0\right\} \subset \mathbb{P}^{2}
$$

Denote by $\bar{X}_{\mathrm{a}}$ the closure of the affine curve $X_{\mathrm{a}}$ in the total space of the line bundle $\mathcal{O}_{\mathbb{P}^{1}}(1)$. We choose $a_{0} \in \mathbb{C}^{*}$ and $\left(h_{1}, h_{2}, h_{3}, h_{4}, h_{5}\right) \in \mathbb{C}^{5}$ such that $\bar{X}_{\mathrm{a}}-Q$ is smooth, and, at $Q(x(Q)=\infty$ and $y(Q)=0)$, the curve $\bar{X}_{\mathrm{a}}$ is locally a normal crossing of two branches (i.e. $a_{1} \neq a_{2}$ ). Denote by $X$ the smooth compactification of $\bar{X}_{\mathrm{a}}$. The double point $Q \in \bar{X}_{\mathrm{a}}$ gives two smooth points, $P_{1}, P_{2} \in X$, on $\bar{X}_{\mathrm{a}}$. Therefore, $X$ is topologically a 2 -torus. Let $P_{3}=(x=\infty, y=1)$ and $P_{4}=(x=\infty, y=-1)$, the modulus is $\mathrm{m}=P_{1}+P_{2}+P_{3}+P_{4}$ and call $X^{\prime}=X_{\mathfrak{m}}$. The data of $\bar{X}_{\mathrm{a}}$ at infinity are

$$
\mathcal{F}=\left\{\left(\left\{e_{1}, e_{2}, e_{3}, e_{4}\right\}, A_{1}\right),\left(\left\{e_{1}, e_{2}\right\},\left(\begin{array}{cc}
a_{1} & 0 \\
0 & b_{1}
\end{array}\right)\right)\right\}
$$


Singular spectral curves and completely integrable systems

621

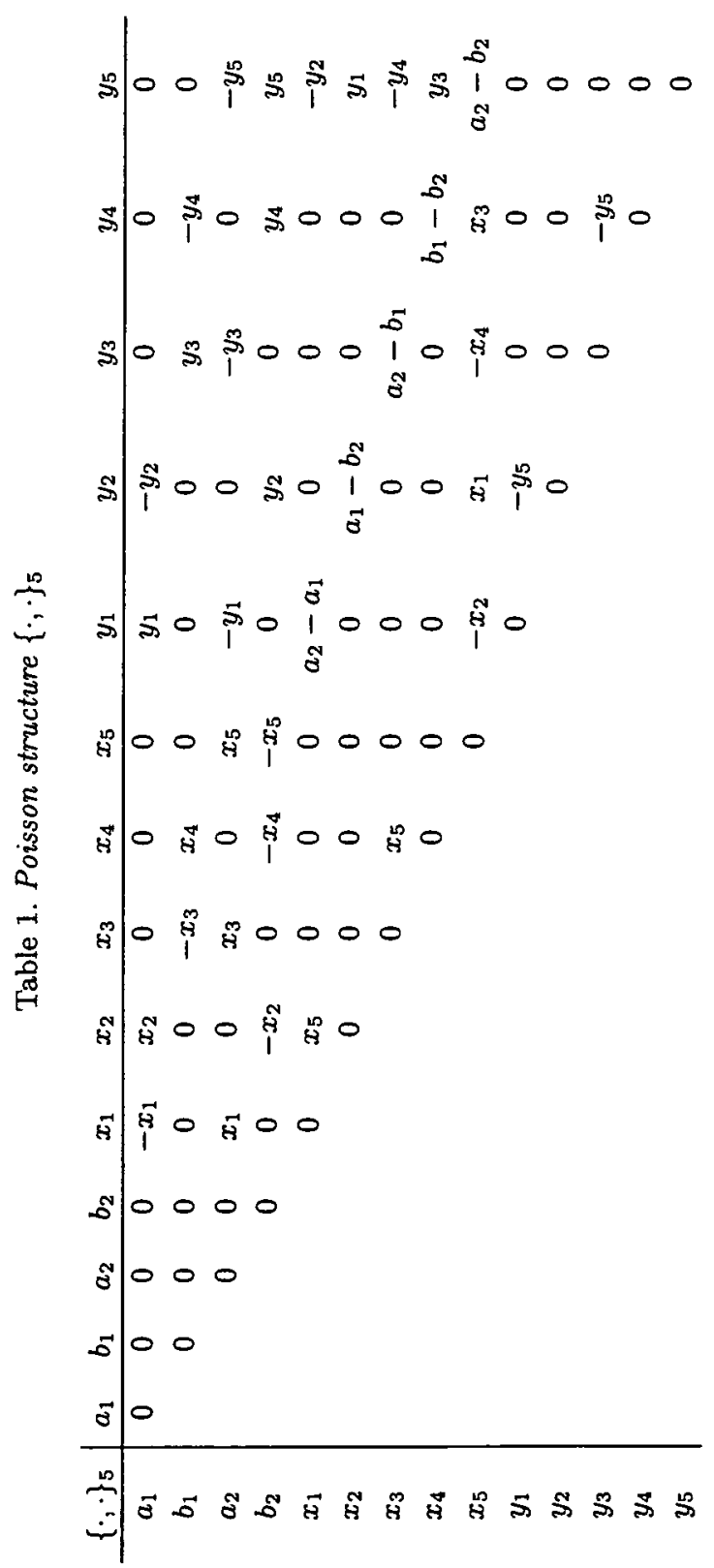

https://doi.org/10.1017/S0013091500021222 Published online by Cambridge University Press 
The isospectral variety $M_{P}^{\mathcal{F}}$ is the set

$$
\left\{\begin{array}{c}
a_{1} a_{2} b_{1} b_{2}-b_{1} b_{2} x_{1} y_{1}-a_{2} b_{1} x_{2} y_{2}-a_{1} b_{2} x_{3} y_{3} \\
-a_{1} a_{2} x_{4} y_{4}-a_{1} b_{1} x_{5} y_{5}+a_{1}\left(x_{3} x_{4} y_{5}+y_{3} y_{4} x_{5}\right) \\
+b_{1}\left(x_{1} x_{2} y_{5}+y_{1} y_{2} x_{5}\right)+\left(x_{1} y_{4}-x_{3} y_{2}\right)\left(x_{4} y_{1}-x_{2} y_{3}\right)=h_{1} \\
a_{1} b_{1}\left(b_{2}-a_{2}\right)+a_{1}\left(x_{3} y_{3}-x_{4} y_{4}\right)+b_{1}\left(x_{1} y_{1}-x_{2} y_{2}\right)=h_{2} \\
\left(x_{i}, y_{i}\right) \in \mathbb{C}^{10}: \quad-a_{1} b_{2}\left(b_{1}+a_{2}\right)-b_{1} a_{2}\left(a_{1}+b_{2}\right)+\left(b_{1}+b_{2}\right) x_{1} y_{1} \\
+\left(b_{1}+a_{2}\right) x_{2} y_{2}+\left(a_{1}+b_{2}\right) x_{3} y_{3}+\left(a_{1}+a_{2}\right) x_{4} y_{4} \\
+\left(b_{1}+a_{1}\right) x_{5} y_{5}-\left(y_{1} y_{2}+y_{3} y_{4}\right) x_{5}-\left(x_{1} x_{2}+x_{3} x_{4}\right) y_{5}=h_{3} \\
\left(a_{1}+b_{1}\right)\left(a_{2}-b_{2}\right)-x_{1} y_{1}+x_{2} y_{2}-x_{3} y_{3}+x_{4} y_{4}=h_{4} \\
a_{1} a_{2}+b_{1} b_{2}+\left(a_{1}+a_{2}\right)\left(b_{1}+b_{2}\right)-x_{1} y_{1} \\
-x_{2} y_{2}-x_{3} y_{3}-x_{4} y_{4}-x_{5} y_{5}=h_{5}
\end{array}\right\} .
$$

Using Theorem 7.2 , we state the result

$$
M_{P}^{\mathcal{F}}=J^{5}\left(X^{\prime}\right)-\Theta^{\prime}
$$

Remark 8.4. Using the same approach, we will be able to construct other completely integrable systems.

Acknowledgements. I express deep gratitude to my supervisor and friend L. Gavrilov, whose guidance and support were important for the successful completion of this project.

\section{References}

1. M. R. Adams, J. Harnad and J. Hurtubise, Isospectral Hamiltonian flows in finite and infinite dimension, Commun. Math. Phys. 134 (1990), 555-585.

2. M. AdLer AND P. VAN MOERBEKE, Linearization of Hamiltonian systems, Jacobi varieties and representation theory, Adv. Math. 38 (1980), 318-379.

3. A. Beauville, Jacobiennes des courbes spectrales et systèmes Hamiltoniens complétement intégrales, Acta. Math. 164 (1990), 211-235.

4. E. D. Belokolos, A. I. Bobenko, V. Z. Enosl'skĭ, A. R. Its and V. B. Matveev, Algebro-geometric approach to nonlinear integrable equations (Springer, 1994).

5. A. I. Bobenko, A. G. Reyman, M. A. Semenov and T. Shansky, The Kowalewski top 99 years later: a Lax pair, generalzations and explicit solutions, Commun. Math. Phys. 122 (1989), 312-354.

6. F. Bottacin, Symplectic geometry on moduli spaces of stable pairs, Ann. Sci. Ecole Norm. Sup. 28 (1995), 391-433.

7. R. DONAGI AND E. MARKMAN, Spectral covers, algebraically completely integrable, Hamiltonian systems and moduli of bundles, Lectures Notes in Mathematics, vol. 1620 (Springer, 1993).

8. L. GAVRILOV, Generalized Jacobians of spectral curves and completely integrable systems, Math. Z. 230 (1999), 487-508. 
9. N. Hitchin, Stable bundles and integrable systems, Duke Math. J. 54 (1987), 91-114.

10. C. JACOBI, Vorlesungen über Dynamik (G. Reimer, Berlin, 1891). (Réimpression: Chelsea, New York, 1967.)

11. S. LANG, Unramified class field theory over function fields in several variables, Ann. Math. 64 (1956), 285-325.

12. S. LANG, Sur les séries $L$ d'une variété algébrique, Bull. Soc. Math. France 84 (1956), 385-407.

13. E. Markman, Spectral curves and integrable systems, Compositio Math. 93 (1994), 255290.

14. A. G. Reyman, M. A. Semenov and T. Shansky, Group theoretical methods in the theory of finite dimensional integrable systems, in Dynamical systems, vol. VII, Encyclopedia of Mathematical Sciences, vol. 16 (Springer, 1994).

15. M. Rosenlicht, Generalized Jacobian varieties, Ann. Math. 59 (1954), 505-530.

16. M. RosenLICHT, A universal mapping property of generalized Jacobian varieties, Ann. Maths 66 (1957), 80-88.

17. J. P. Serre, Groupes algébriques et corps de classes (Hermann, Paris, 1959).

18. P. VAN MOERBEKE AND D. MUMFORD, The spectrum of difference operators and algebraic curves, Acta Math. 143 (1979), 93-154.

19. O. Vivolo, Systèmes intégrables et courbes algébriques, $\mathrm{PhD}$ thesis, Université de Toulouse III (1997).

20. A. WEIL, Variétés abéliennes et courbes algébrique (Hermann, Paris, 1948). 